\title{
KM3NeT: Status and Prospects for Neutrino Astronomy at Low and High Energies
}

\author{
Feifei Huang* on behalf of the KM3NeT collaboration \\ IN2P3, IPHC, 23 rue du Loess, Strasbourg, 67037, France \\ Aix Marseille Univ, CNRS/IN2P3, CPPM, Marseille, France \\ E-mail: feifei.huang@cppm.in2p3.fr
}

\begin{abstract}
$\mathrm{KM} 3 \mathrm{NeT}$ is a multi-purpose cubic-kilometer neutrino observatory currently being deployed at the bottom of the Mediterranean Sea. It consists of two detectors: ORCA and ARCA (for Oscillation and Astroparticle Research with Cosmics in the Abyss, respectively). ARCA will instrument 1 Gton of seawater, with the primary goal of detecting cosmic neutrinos with energies between several tens of $\mathrm{GeV}$ and PeV. Due to its position in the Northern Hemisphere, ARCA will provide an optimal view of the Southern sky including the Galactic Center. ORCA is a smaller (few Mtons) and denser array, optimized for the detection of atmospheric neutrinos in the $1-100 \mathrm{GeV}$ range. It can also investigate low-energy neutrino astronomy, such as $\mathrm{MeV}$-scale core-collapse supernova. This proceeding presents the current status of the KM3NeT infrastructure, its outlook on neutrino astronomy, and its multi-messenger program status.
\end{abstract}

40th International Conference on High Energy physics - ICHEP2020

July 28 - August 6, 2020

Prague, Czech Republic (virtual meeting)

\footnotetext{
${ }^{*}$ Speaker
} 


\section{Introduction}

Being neutral and only weakly interacting, neutrinos do not get deflected or absorbed during their intergalactic travel, pointing back to the sources where they are created. This makes them excellent tools for studying our universe, especially when combined with other cosmic messengers. KM3NeT, the cubic-kilometer neutrino observatory under construction in the Mediterranean Sea, will be able to detect neutrinos in an energy range from $\mathrm{MeV}$-scale core-collapse supernova neutrinos (CCSNs), to atmospheric neutrinos in the $\mathrm{GeV}$ range, to the most energetic astrophysical neutrinos from $\mathrm{TeV}$ up to a few PeV. This proceeding reviews KM3NeT's science prospects for neutrino astronomy, the detector status, and newest results.

\subsection{The KM3NeT Detector}

The KM3NeT infrastructure consists of building blocks, each a 3D array of digital optical modules (DOMs). A block comprises 115 vertical strings, each with 18 DOMs. Each DOM has 31 3-inch PMTs.

A phased strategy is used for detector implementation [1]. At Phase-2, KM3NeT will have two detectors ORCA and ARCA. ORCA consists of one building block with its geometry optimized for detecting atmospheric neutrinos. ARCA consists of two blocks aiming at astrophysical neutrinos. The current KM3NeT detectors have one string of ARCA and six strings of ORCA (Phase-1).

The PMTs detect the Cherenkov light emitted by secondary particles from neutrino interactions in seawater. The PMT analog signals passing a preset threshold are digitized and sent to shore where online data filtering is performed and the physics events are filtered from background.

\subsection{Event Signatures, Reconstruction Resolution}

Two types of event signatures are observed in the detector: 1) track-like events with a visible track left by the daughter muon of the charged-current interactions of muon neutrinos; 2) showerlike events that have no visible muon track. The track-like events give great directional resolution, which is vital for point-like neutrino source search. Fig.1 shows the angular resolution of ARCA, with an excellent angular resolution of better than $0.1^{\circ}$ above $100 \mathrm{TeV}$. ARCA shower-like events' median angular resolution will be around $2^{\circ}$.

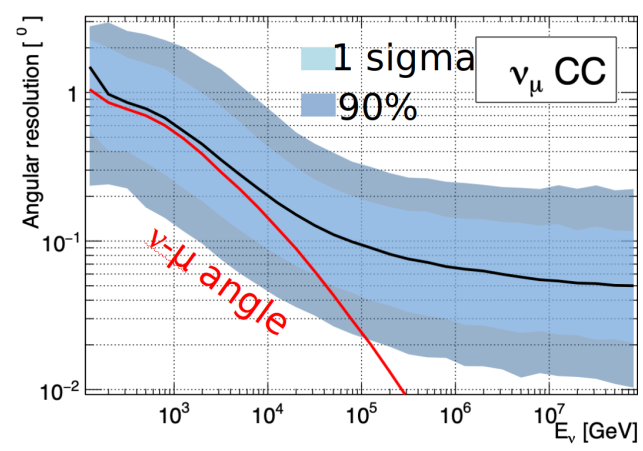

Figure 1: Angular resolution of ARCA. Black line is the median error of angle between the reconstructed track and true neutrino [2].

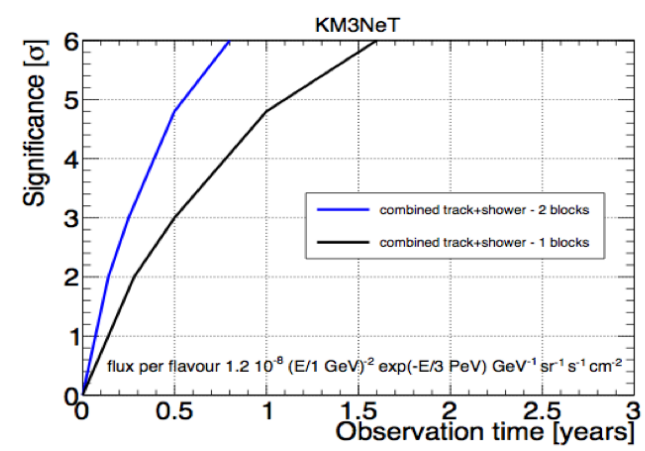

Figure 2: ARCA sensitivity of diffuse astrophysical neutrinos from an assumed flux, with a combined track and shower event selection [1]. 


\section{Diffuse Neutrino Flux}

The observation of diffuse astrophysical neutrinos in 2013 [3] is a milestone in neutrino astronomy. Fig.2 shows the sensitivity of ARCA to diffuse astrophysical neutrinos, assuming they are isotropic, flavor-symmetric, and following a power-law spectrum with a cut-off at a few PeV. It takes only one year with one block of ARCA to reach a $5 \sigma$ observation, and 0.5 years with full ARCA. See more details in Ref.[1].

\section{Point-like Neutrino Sources}

Using a generic $E^{-2}$ neutrino flux as approximation, the ARCA sensitivity towards extragalactic neutrino sources is evaluated [4]. Located in the Northern Hemisphere, ARCA will have full sky coverage and good sensitivity to sources in the Southern Hemisphere, where it will perform over one order of magnitude better than IceCube, shown in Fig.3 (left).

For the study of galactic neutrino sources, with the neutrino flux derived from their gamma-ray flux, assuming $100 \%$ hadronic gamma-ray emission, they can be observed by ARCA in a few years (Fig.3 (right)). For example, Supernova Remnant Vela Jr can be observed with a $3 \sigma$ significance within 6 years, RX J1713.7-3946 within 5.5 years. See more results and details in Ref.[4].
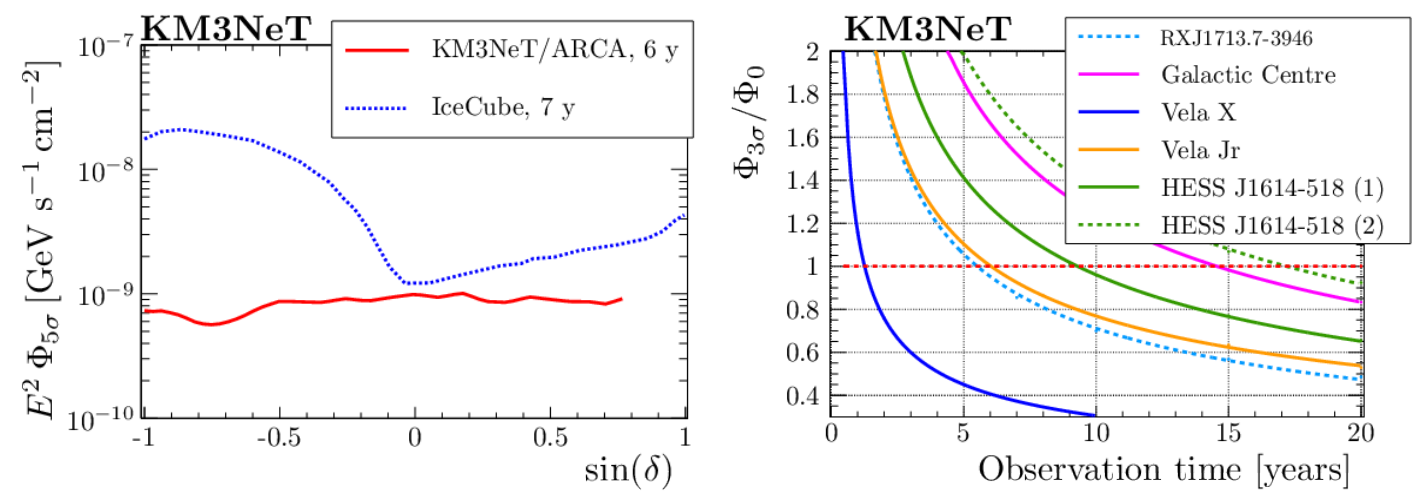

Figure 3: KM3NeT sensitivity to approximated extra-galactic neutrino flux and extended galactic sources [4]. Left: Discovery flux vs. source declination with six years of ARCA (red), comparing with IceCube (blue) with an equivalent exposure. Right: Ratio of the flux for $3 \sigma$ discovery potential $\phi_{3 \sigma}$ to the expectation flux $\phi_{v}$ vs. observation time for different sources shown in different colors.

\section{Core-Collapse Supernova Neutrino}

Core-collapse supernova neutrinos will arrive on the Earth before the electromagnetic signals. Detection of these neutrinos is crucial in understanding the explosion mechanism and vital for an early warning to direct optical telescopes for the observation of the next Galactic supernova.

In the event of a CCSN burst, the MeV-scale energy of the supernova neutrinos is too low to generate coincident light in multiple DOMs in KM3NeT, but the large amount of them (can be up to thousands) will result in higher counting rates of individual PMTs and an increase of the number of coincident hit PMTs within a DOM (multiplicity). 
Fig.4 (left) [5] shows the expected event rates from neutrino bursts of three simulated CCSN progenitors and background rates (mainly from ${ }^{40} \mathrm{~K}$ decays and bioluminescence). The KM3NeT supernova monitoring system evaluates the number of coincident PMTs every $100 \mathrm{~ms}$ in a $500 \mathrm{~ms}$ sliding window. It is already connected to SNEWS (SuperNova Early Warning System), sending alerts with false alarm rate less than 1/week. The MeV CCSN neutrino monitoring is one of the two pipelines of the KM3NeT real-time alert program. The other pipeline is for the GeV-PeV neutrinos (as energy is large enough to hit several DOMs coincidentally) and is discussed in the next section.

Fig.4 (right) [5] shows KM3NeT's expected CCSN sensitivities as a function of distance. Over $95 \%$ of Galactic CCSN progenitors are covered by KM3NeT at $5 \sigma$ discovery potential.

Apart from MeV-scale CCSN, more low energy astrophysical neutrino searches with ORCA can be found in Ref.[6].
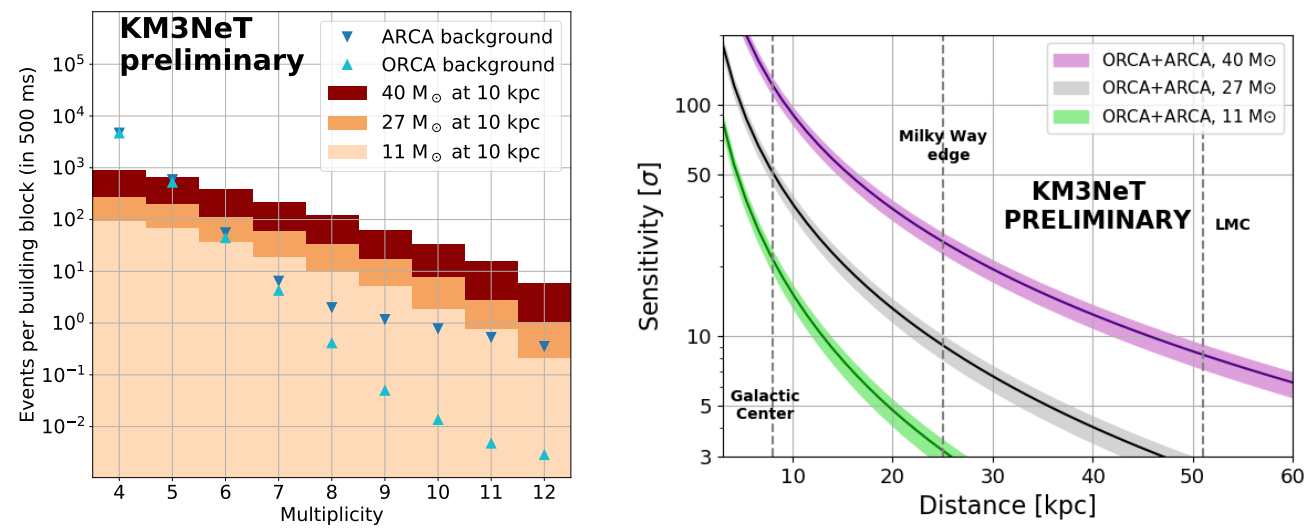

Figure 4: Left: Supernova events expected at different multiplicity values compared with background. Right: KM3NeT Sensitivity of CCSN as a function of distance, based on 7-11 multiplicity coincidences in the $500 \mathrm{~ms}$ time window [5].

\section{Real-time Alert Analysis Framework}

The goals of the real-time $(\mathrm{GeV}-\mathrm{PeV})$ neutrino alert pipeline are: 1) receive external electromagnetic, gravitational wave, or neutrino alerts and perform online neutrino correlation search, 2) send all-flavor, all-sky neutrino real-time alerts to external observatories for follow-up. This entails a fast online reconstruction and a fast selection of a high-purity neutrino sample.

This pipeline will take the physics events produced by online data filtering [1] and perform online event reconstruction, event classification, neutrino correlation search, alert receiving and sending applications, etc. Among them, the event reconstruction and the track event classification are running online with the current 6-string ORCA detector. The online reconstruction uses the same reconstruction algorithms [7] as in offline processing, but does not run the complete reconstruction chain for every event.

With the 6-string ORCA detector, the track direction reconstruction takes $0.1 \mathrm{~s}$ per event on average (currently online), the shower reconstruction takes 1s (currently offline, with possible parameter optimization to be studied), and the online classification $0.01 \mathrm{~s}$. The combined elapsed time for online data filtering, track reconstruction, and classification is $4 \mathrm{~s}$ on average. 
Fig. 5 shows the angular resolution and effective area with preliminary selections based on the track classification. Further optimizations, the training of shower classifiers, and the development of the other modules are ongoing.
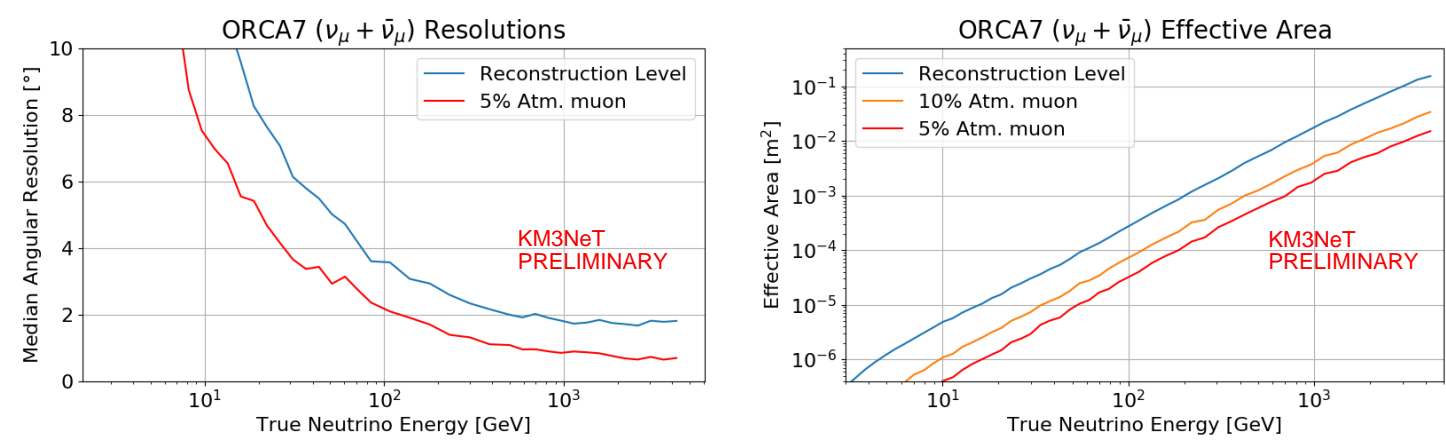

Figure 5: Angular resolution (left) and effective area (right) comparisons for preliminary selections and the reconstruction level with 7-string ORCA MC [8].

\section{Latest Results and Outlook}

ORCA has been operating with four strings from July 2019 to January 2020, and with six strings since January 2020. ARCA currently has one string and was operational with two strings from May 2016 to April 2017. This section reports the latest results with these first few strings.

Fig.6 (left) shows an event selection of upgoing track-like events with 4-string ORCA data in 133.1 days of livetime, with a 99\% purity and an event rate of 2 to 3 per day. Fig.6 (right) shows a refined selection where oscillation effects can be seen at a $2 \sigma$ significance [9].

Fig. 7 shows the measurement of the atmospheric muon flux [10] between 2232-3386 m seawater depth using 1-string ORCA and 2-string ARCA data. The measurements are found to be compatible with the Bugaev parametrization and with the ANTARES results (Fig.7). This verifies the detector calibration technique, proving that we have a good understanding of our detectors.
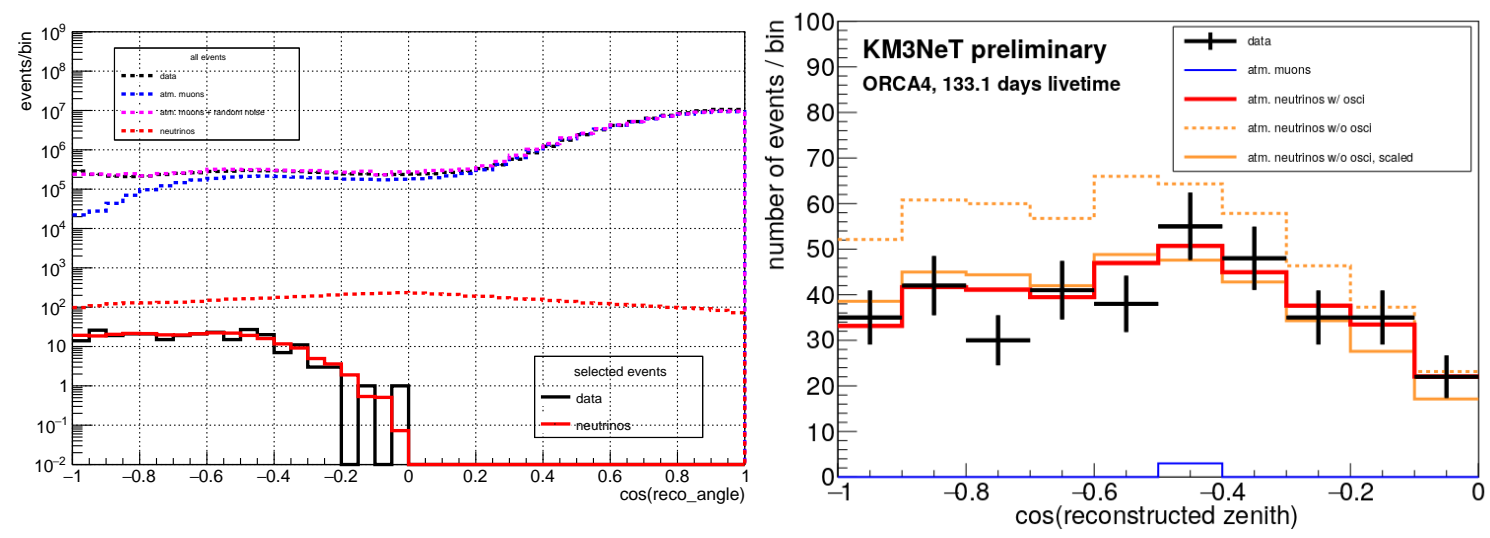

Figure 6: Preliminary selection with 4-string ORCA data [9]. Left: event distributions before and after the upgoing track selection. Right: zenith distributions for oscillation studies, dashed line is the no-oscillation hypothesis. 


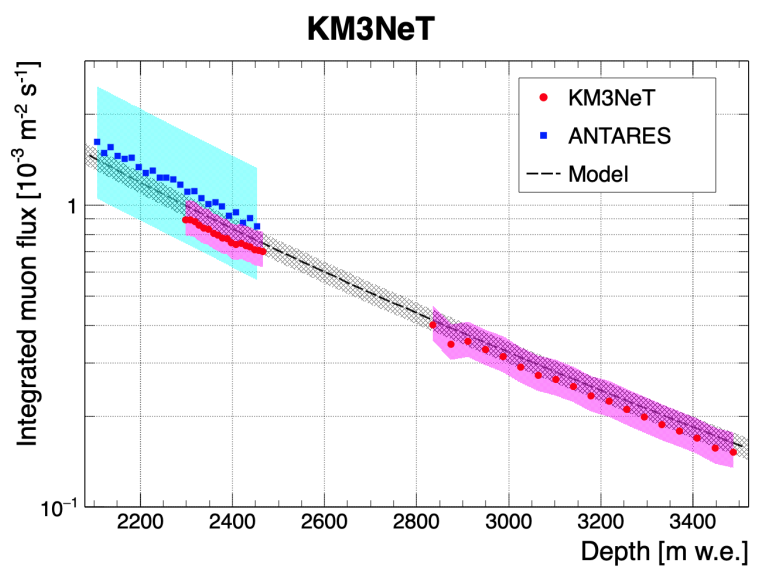

Figure 7: Atmospheric muon fluxes vs. sea water depth. Red (blue) dots and bands are the measurements by KM3NeT (ANTARES) and their corresponding systematic errors [10].

\section{Summary}

With an excellent angular resolution $\left(<0.1^{\circ}\right.$ above $100 \mathrm{TeV}$ with track events), a large neutrino sky coverage, and the Galactic center in view, KM3NeT will be a key contributor to neutrino astronomy - both at the highest energies (TeV-PeV astrophysical neutrinos) and at the low energies (MeV-scale CCSN bursts). The latest results with the first few strings prove that the detectors are well understood, giving confidence to the future progress of KM3NeT and its scientific potentials.

\section{References}

[1] S Adrián-Martínez, et al. (KM3NeT Coll.), Journal of Physics G: Nuclear and Particle Physics, 43(8):084001, June 2016

[2] D. Samtleben (KM3NeT Coll.), Neutrino2020, http://doi.org/10.5281/zenodo.4124368

[3] Aartsen MG, et al., (IceCube Coll.), Science, 342(6161), 1242856, Nov 2013

[4] S. Aiello, et al., (KM3NeT Coll.), Astropart. Phys. 111, 100-110 (2019)

[5] A. Coleiro, M. Colomer, D. Dornic, V. Kulikovskiy, M. Lincetto, (KM3NeT Coll.), Neutrino2020 (poster \#245)

[6] G. de Wasseige1, A. Kouchner, M. Colomer, D. Dornic, S. Hallmann, (KM3NeT Coll.) PoS (ICRC2019) 934

[7] K. Melis, A. Heijboer, M. De Jong, (KM3NeT Coll.), PoS (ICRC2017) 950

[8] D. Dornic, F. Huang, M. Lincetto (KM3NeT Coll.), Neutrino2020 (poster \#340)

[9] D. Stavropoulos, J. Hofestädt, L.A. Fusco (KM3NeT Coll.), Neutrino2020 (poster \#363)

[10] M. Ageron, et al., (KM3NeT Coll.), The European Physical Journal C 80, 99 (2020) 